EPJ Web of Conferences 21, 10003 (2012)

DOI: $10.1051 /$ epjconf/20122110003

(c) Owned by the authors, published by EDP Sciences, 2012

\title{
Bound state densities and the Helmholtz free energy
}

\author{
F. T. Dalmolin ${ }^{1, \text { a }}$, M. Dutra ${ }^{1}$, B. V. Carlson ${ }^{1}$, R. Donangelo ${ }^{2,3}$, and S. R. Souza ${ }^{2,4}$ \\ 1 Instituto Tecnológico de Aeronáutica, São José dos Campos SP, Brazil \\ 2 Instituto de Física, Universidade Federal do Rio de Janeiro, Rio de Janeiro, Brazil \\ 3 Instituto de Física, Universidad de la República, Montevideo, Uruguay \\ 4 Instituto de Física, Universidade Federal do Rio Grande do Sul, Porto Alegre, Brazil
}

\begin{abstract}
Bohr's conception of the compound nucleus is based on the idea of 'longlived' nuclear states in which all single particles are bound. We briefly discuss the properties of the density of bound states and then use two prescriptions, that of Brack and Quentin and that of Bonche, Levit e Vautherin to calculate the equivalent temperaturedependent quantity - the Helmholtz free energy. We compare the temperature dependence of the latter, as well as that of the excitation energy and entropy, obtained using the two prescriptions in self-consistent calculations within the relativistic Hartree and Skyrme models. We then discuss the extended, temperature-dependent liquid-drop approximation to the excitation and free energies obtained from fits to the self-consistent calculations over a wide range of charge and mass numbers.
\end{abstract}

\section{Introduction}

The statistical decay of the compound nucleus is determined by the squared matrix element of the transition to the continuum and by the density of final states. In the usual Hauser-Feshbach and WeisskopfEwing evaporation models, the transition matrix element is expressed in terms of the transmission coefficients or the cross section of the inverse formation process. In the Fermi breakup and statistical multifragmentation models, the transition matrix element is taken to be equal for all decay channels. In all cases the density of quasi-bound final fragment states plays an important role.

The density of quasi-bound states of a nucleus is the density of states in which all neutrons are in bound single-particle states and all protons are in single particle states that are either bound or in long-lived single particle states well below the Coulomb barrier. We associate these states with the long-lived states of Bohr's conception of the compound nucleus [1]. The excitation energy dependence of the density of states was first estimated by Bethe [2] and has been the center of a great deal of theoretical and experimental effort [3-5]. All of these calculations of the density of quasi-bound states begin with a static set of single-particle states and analyze their occupation as a function of the temperature. The Helmholtz free energy determined in this manner, $F^{*}(T)$ is related to the density of states $\omega\left(E^{*}\right)$ by a Laplace transform,

$$
e^{-F^{*}(T) / T}=\int_{0}^{\infty} e^{-E^{*} / T} \omega\left(E^{*}\right) d E^{*}
$$

The Laplace transformation is then inverted to determine the density of states.

However, as a nucleus is heated, it expands and becomes less bound. At a sufficiently high temperature, it will evaporate completely. These effects are not taken into account when a static set of

a FTD and MD are supported by FAPESP. BVC acknowledges support from FAPESP and the CNPq. e-mail: brett@ita.br

This is an Open Access article distributed under the terms of the Creative Commons Attribution-Noncommercial License 3.0, which permits unrestricted use, distribution, and reproduction in any noncommercial medium, provided the original work is properly cited. 


\section{EPJ Web of Conferences}

single-particle levels are used to calculate the density, but can be estimated from self-consistent calculations of hot nuclei. Here we take two approaches to such calculations. In the simplest, we perform temperature-dependent self-consistent mean field calculations restricted to the states that are quasibound at the given temperature. Similar calculations were performed years ago by Brack and Quentin (BQ) [6,7], Typical results of this type of calculation are shown in the Fig. 1, for the case of ${ }^{56} \mathrm{Fe}$. As the temperature increases, the nucleus expands and decreases in density.

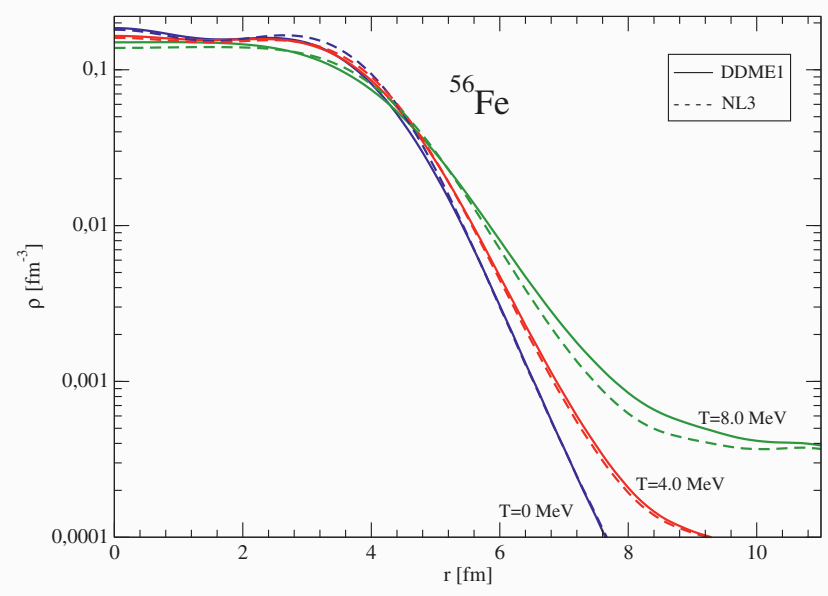

Fig. 1. Nucleon density using the BQ prescription with the NL3 and DDME1 parametrizations of the relativistic Hartree approximation.

Another approach, developed by Bonche, Levit and Vautherin, (BLV) [8,9] takes into account all single-particle levels of a heated nucleus in the self-consistency calculation. To extract the contribution of the quasi-bound nucleons alone, the contribution of the gas of unbound nucleons must be subtracted. This is done by performing two self-consistent calculations - one of the nucleus plus gas and another of the gas alone (both with identical Fermi energies) and subtracting extensive quantities (entropy, excitation energy, baryon density) obtained for the latter from those of the former. Typical results from this type of calculation are shown in Figs. 2 and 3. Again, as the temperature increases, the nucleus expands and decreases in density. The density of the gas is zero at zero temperature and increases with temperature until the nucleus disappears completely, typically at a temperature of about $12 \mathrm{MeV}$.

We have performed calculations using both the BQ and the BLV prescriptions in the self-consistent relativistic Hartree approximation using the nonlinear NL3 [10] and density-dependent DDME1 [11] parameter sets. To our knowledge, these sets provide the best agreement with ground state nuclear masses obtained using the relativistic Hartree approximation. We have also performed calculations using the nonrelativistic BSk14 [12] and NRAPR [13] Skyrme interactions in the Thomas-Fermi approximation to the BLV prescription. These Skyrme parametrizations furnish a good description of ground state nuclear masses as well as the the properties of infinite nuclear matter at saturation density. The BLV results are quite similar in all cases, as can be seen in Figs 2 and 3.

\section{Thermodynamic quantities}

The extensive quantities that can be obtained from the self-consistent calculations are the entropy, the excitation energy, the baryon and charge density and other density-related quantities, such as defor- 


\section{$\mathrm{CNR} * 11$}

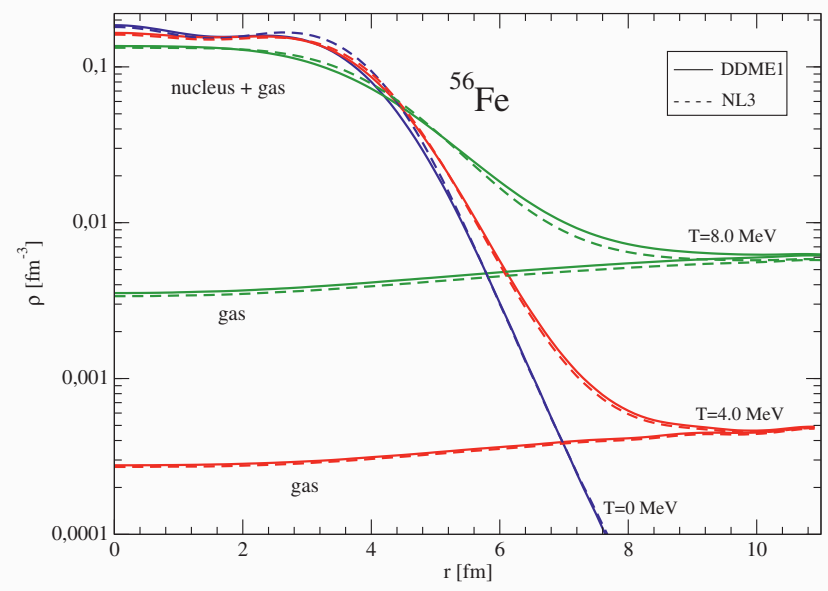

Fig. 2. Nucleon density using the BLV prescription with the NL3 and DDME1 parametrizations of the relativistic Hartree approximation.

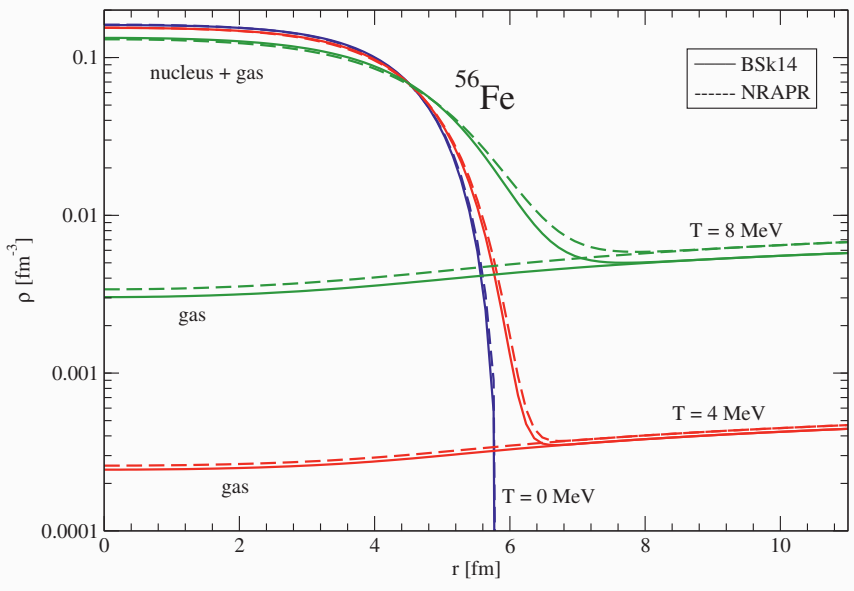

Fig. 3. Nucleon density using the BLV prescription with the BSk14 and NRAPR Skyrme parametrizations of the Thomas-Fermi approximation.

mations and rms radii. Deformation and pairing are found to vanish at extremely low temperatures. Normal isovector pairing usually disappears at temperatures below $1 \mathrm{MeV}$ and almost all calculations yield nuclei that are spherical at $2 \mathrm{MeV}$ [14].

The entropy and excitation energy are related to the Helmholtz free energy through

$$
S^{*}=-\frac{\partial F^{*}}{\partial T}
$$




\section{EPJ Web of Conferences}

and

$$
E^{*}=F^{*}-T \frac{\partial F^{*}}{\partial T}
$$

The entropy $S^{*}(T)$ can be determined directly from the calculations, while the excitation energy is obtained as the difference between the binding energy $E_{b n d}(T)$ at a temperature $T$ and that at temperature zero,

$$
E^{*}(T)=E_{b n d}(T)-E_{b n d}(0) \text {. }
$$

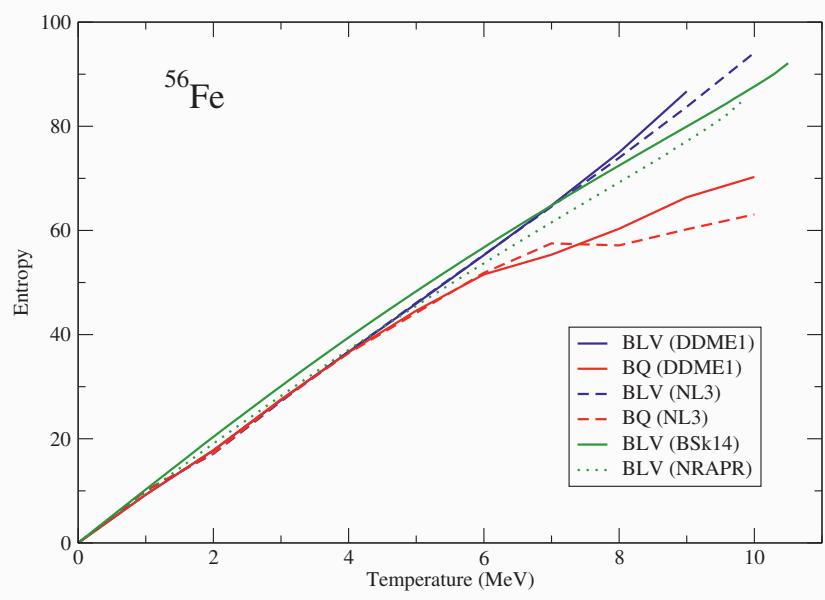

Fig. 4. Entropy of ${ }^{56} \mathrm{Fe}$ as a function of temperature.

In Fig. 4, we display the entropy obtained for the calculations in the case of ${ }^{56} \mathrm{Fe}$. All calculations furnish similar values at temperatures below about $6 \mathrm{MeV}$. Above that value, the calculations using only the bound single-particle states display a deviation from the nearly linearly increasing entropy of the BLV prescription.

In Fig. 5, we show the excitation energy obtained different calculations in the case of ${ }^{56} \mathrm{Fe}$. Again, all calculations furnish similar values at temperatures below about $6 \mathrm{MeV}$. Above this value, the calculations using only the bound single-particle states again deviate from the quadratically-increasing excitation energy of the BLV calculations.

Despite the very similar behavior of the entropy and excitation energy of the two sets of calculations below about $6 \mathrm{MeV}$, we see in Fig. 6 that their rms radii are very different. The rms radius increases almost linearly in the calculations including only bound single-particle states while it increases quadratically and, except at very high temperatures, remains smaller in the case in which the nucleus is in equilibrium with a surrounding gas. We interpret the smaller radius of the calculations with the BLV prescription as an effect of the pressure exerted by the gas on the hot nucleus. The smaller radii obtained using the Skyrme interactions could be an artefact of the Thomas-Fermi approximation, for which no wave functions and thus no exponentially decaying tails exist. We plan to test this hypothesis in the future with Skyrme Hartee-Fock calculations. 


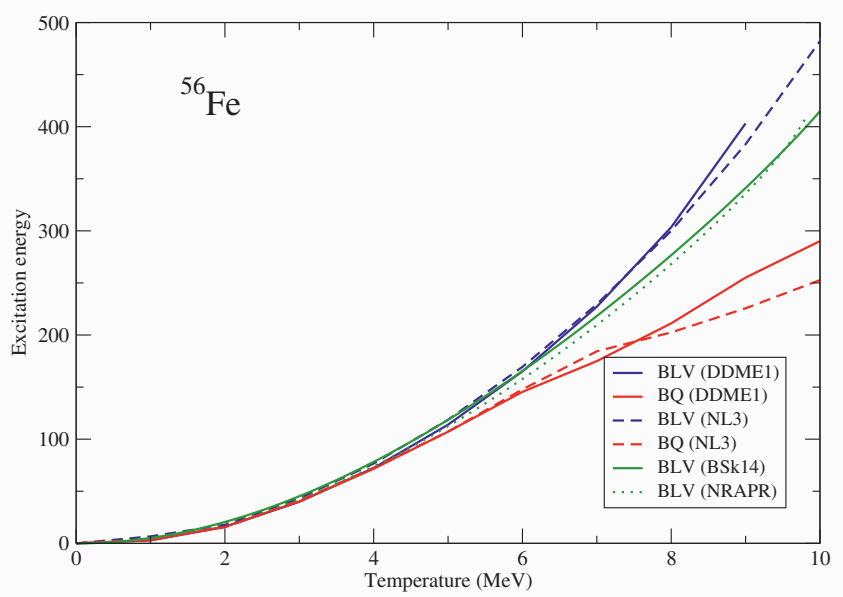

Fig. 5. Excitation energy of ${ }^{56} \mathrm{Fe}$ as a function of temperature.

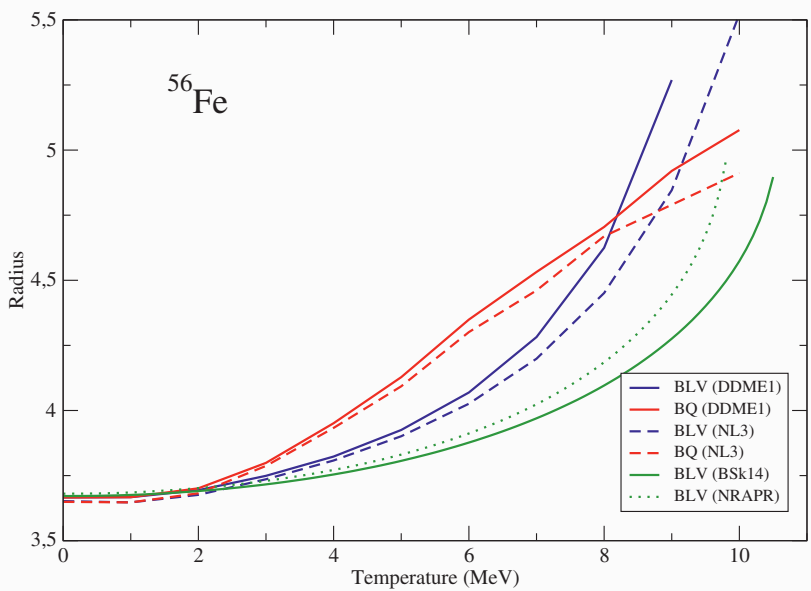

Fig. 6. Rms radius of ${ }^{56} \mathrm{Fe}$ as a function of temperature.

\section{Parametrization of the free energy}

One of the goals of this study is to obtain a consistent global parametrization of the Helmholtz free energy from the calculations described here. With this in mind, we have calculated the statistical properties of a large set of nuclei up to the temperature at which they no longer exist and have used these results to perform global fits to an extended temperature-dependent liquid-drop expression similar to that used in statistical multifragmentation models [15]. We take 


$$
\begin{aligned}
F(T)= & c_{1} A+c_{2} A^{2 / 3}+c_{4} d^{2} A+c_{5} A^{1 / 3}+c_{6} \frac{Z(Z-1)}{A^{1 / 3}} \\
& -\left(c_{7} A+c_{8} A^{2 / 3}+c_{9} d^{2} A\right) T^{2},
\end{aligned}
$$

where $d=(1-2 Z / A) /\left(1+c_{3} A^{-1 / 3}\right)$. We use a simple $T^{2}$ parametrization of the excitation-dependent terms as such an expression is consistent with our results for the entropy and excitation energy up to a temperature of at least $6 \mathrm{MeV}$. We adjust the parameters of the the free energy to the binding energies and entropies obtained for 189 nuclei in the range $2 \leq Z \leq 82$ and $4 \leq A \leq 250$. The fit to the relativistic Hartree calculations was performed using calculation at temperatures from $1 \mathrm{Mev}$ to 6 $\mathrm{MeV}$, to avoid the effects of pairing at low temperature. The Skyrme-Thomas-Fermi calulations from a temperature of $0 \mathrm{MeV}$ to $6 \mathrm{MeV}$ were used in their fits.

Table 1. Parameters of the temperature-independent part of the Helmholtz free energy.

\begin{tabular}{|c|c|c|c|c|c|c|}
\hline Model & $\begin{array}{c}c_{1} \\
(\mathrm{MeV})\end{array}$ & $\begin{array}{c}c_{2} \\
(\mathrm{MeV})\end{array}$ & $c_{3}$ & $\begin{array}{c}c_{4} \\
(\mathrm{MeV})\end{array}$ & $\begin{array}{c}c_{5} \\
(\mathrm{MeV})\end{array}$ & $\begin{array}{c}c_{6} \\
(\mathrm{MeV})\end{array}$ \\
\hline \hline BQ-NL3 & -14.22 & 11.07 & 1.113 & 29.69 & 10.19 & 0.624 \\
\hline BQ-DDME1 & -15.34 & 16.60 & 1.609 & 37.85 & 3.92 & 0.679 \\
\hline BLV-NL3 & -13.99 & 10.36 & 1.198 & 29.61 & 9.83 & 0.603 \\
\hline BLV-DDME1 & -14.88 & 15.01 & 1.114 & 31.12 & 3.42 & 0.649 \\
\hline BSk14 & -14.72 & 11.70 & 0.685 & 27.56 & 7.10 & 0.665 \\
\hline NRAPR & -14.40 & 10.14 & 0.753 & 28.61 & 9.07 & 0.652 \\
\hline
\end{tabular}

The temperature-independent parameters obtained from the fits are given in the table below. In principle, these parameters describe the ground state nuclear energies. However, as they are obtained from a fit that includes the temperature dependence, their values are shifted slightly from those that would be obtained from a fit of the zero temperature values alone, to compensate inadequacies of the functional form assumed. The symmetry and Coulomb coefficients, $c_{4}$ and $c_{6}$ are in fact close to commonly used values of $c_{4} \approx 30 \mathrm{MeV}$ and $c_{6} \approx 0.72 \mathrm{MeV}$. However, the volume and surface terms are smaller than their usual values of $c_{1} \approx-16 \mathrm{MeV}$ and $c_{2} \approx 18 \mathrm{MeV}$, respectively.

Table 2. Parameters of the temperature-dependent part of the Helmholtz free energy.

\begin{tabular}{|c|c|c|c|}
\hline Model & $\begin{array}{c}c_{7} \\
(\mathrm{MeV})\end{array}$ & $\begin{array}{c}c_{8} \\
(\mathrm{MeV})\end{array}$ & $\begin{array}{c}c_{9} \\
(\mathrm{MeV})\end{array}$ \\
\hline \hline BQ-NL3 & 0.091 & -0.063 & -0.541 \\
\hline BQ-DDME1 & 0.090 & -0.069 & -0.623 \\
\hline BLV-NL3 & 0.059 & 0.091 & -0.070 \\
\hline BLV-DDME1 & 0.058 & 0.094 & -0.107 \\
\hline BSk14 & 0.064 & 0.080 & -0.085 \\
\hline NRAPR & 0.057 & 0.089 & -0.088 \\
\hline
\end{tabular}

Of special interest to us is the temperature dependence of the volume, surface and symmetry terms of the free energy. Our results furnish volume and surface terms slightly smaller in value than the usual ones of $c_{7} \approx 0.0625$ and $c_{8} \approx 0.139$ when the BLV prescription is used. The coefficients are very different when the BQ prescription is used, with the surface coefficient of opposite sign. We find that the symmetry term, which is usually considered to be independent of the temperature, in fact varies about as strongly with temperature as does the surface term, in the BLV case, and much 


\section{$\mathrm{CNR} * 11$}

more strongly in the BQ prescription. Such a variation could have important effects on the isotopic distribution of fragments emitted at high excitation energy, a possibility that we are now studying in the statistical multifragmentation model [15]. We are also studying the significance of the differences of the thermodynamic quantities in the BLV and BC representations and in their parametrizations.

\section{References}

1. N. Bohr, Nature 137 (1936) 344.

2. H. A. Bethe, Rev. Mod. Phys. 9 (1937) 69.

3. C. Bloch, Phys. Rev. 93 (1954) 1094.

4. C. Bloch, Statistical Nuclear Theory, Les Houches Lectures (1968) 305.

5. A. Bohr and B.R. Mottelson, Nuclear Structure, vol. 1 (Benjamin, Reading, Mass., 1969) p.281

6. M. Brack and Ph. Quentin, Phys. Lett. 52B (1974) 159.

7. M. Brack and Ph. Quentin, Physica Scripta A10 (1974) 163.

8. P. Bonche, S. Levit and D. Vautherin, Nuclear Physics A427 (1984) 278.

9. P. Bonche, S. Levit and D. Vautherin, Nucl. Phys. A436 (1985) 265.

10. G. A. Lalazissis, J. König and P. Ring, Phys. Rev. C 55 (1997) 540.

11. T. Niksic, D. Vretenar, P. Finelli, and P. Ring, Phys. Rev. C 66 (2002) 024306.

12. S. Goriely, M. Samyn, and J. Pearson, Phys. Rev. C 75 (2007) 064312.

13. A. W. Steiner, M. Prakash, J. M. Lattimer, and P. J. Ellis, Phys. Rep. 411 (2005) 325.

14. R. Lisboa, M. Malheiro, and B. V. Carlson, IJMP 16 (2007) 3032.

15. W. P. Tan, S. R. Souza, R. J. Charity, R. Donangelo, W. G. Lynch, and M. B. Tsang, Phys. Rev. C 68 (2003) 034609. 